\title{
A Reinforcement Learning-driven Translation Model for Search-Oriented Conversational Systems
}

\author{
Wafa Aissa \\ Sorbonne Université \\ CNRS, LIP6 \\ F-75005 Paris, France \\ wafa.aissaelip6.fr
}

\author{
Laure Soulier \\ Sorbonne Université \\ CNRS, LIP6 \\ F-75005 Paris, France \\ laure.soulierdip6.fr
}

\author{
Ludovic Denoyer \\ Sorbonne Université \\ CNRS, LIP6 \\ F-75005 Paris, France \\ ludovic.denoyeralip6.fr
}

\begin{abstract}
Search-oriented conversational systems rely on information needs expressed in natural language (NL). We focus here on the understanding of NL expressions for building keywordbased queries. We propose a reinforcementlearning-driven translation model framework able to 1) learn the translation from NL expressions to queries in a supervised way, and, 2) to overcome the lack of large-scale dataset by framing the translation model as a word selection approach and injecting relevance feedback as a reward in the learning process. Experiments are carried out on two TREC datasets. We outline the effectiveness of our approach.
\end{abstract}

\section{Introduction}

Artificial Intelligence, and more particularly deep learning, have recently opened tremendous perspectives for reasoning over semantics in textbased applications such as machine translation (Lample et al., 2017), chat-bot (Bordes and Weston, 2016), knowledge base completion (Lin et al., 2015) or extraction (Hoffmann et al., 2011). Very recently, conversational information retrieval (IR) has emerged as a new paradigm in IR (Burtsev et al., 2017; Joho et al., 2018), in which natural conversations between humans and computers are used to satisfy an information need. As for now, conversational systems are limited to simple conversational interactions (namely, chit-chat conversations) (Li et al., 2016; Ritter et al., 2011), closed worlds driven by domain-adapted or slotfilling patterns (Bordes and Weston, 2016; Wang and Lemon, 2013) (e.g., a travel planning task requiring to book a flight, then a hotel, etc...), or knowledge-base extraction (e.g., information extraction tasks) (Dhingra et al., 2017).

In contrast, search-oriented conversational systems (SOCS) aim at finding information in an open world (both unstructured information sources and knowledge-bases) in response to users' information needs expressed in natural language (NL); the latter often being ambiguous. Therefore, one key challenge of SOCS is to understand users' information needs expressed in NL to identify relevant documents.

Formulating an information need through queries has been outlined as a difficult task (Vakulenko et al., 2017; Agichtein et al., 2006; Joachims, 2002) which is generally tackled by refining/reformulating queries using pseudorelevance feedback or users' clicks. In SOCS, there is an upstream challenge dealing with the building of the query from a NL expression that initiates the search session to avoid useless users' interactions with the system. This problem could be tackled for instance through deep neural translation models (e.g., encoder-decoder approaches) as initiated by (Song et al., 2017; Yin et al., 2017). However, these methods learn the query formulation model independently of the search task at hand. To overpass this limitation, (Nogueira and Cho, 2017) have proposed a reinforcement learning model for query reformulation in which the reward is based on terms of documents retrieved by the IR system.

In this work, we propose to bridge these two lines of work: 1) machine translation to learn the mapping between information needs expressed in NL and information needs formulated using keywords (Song et al., 2017; Yin et al., 2017), and 2) reinforcement learning to inject the task objectives within the machine translation model (Nogueira and Cho, 2017). More particularly, we propose a two-step model which first learns the translation model through the supervision of NL-query pairs and then refines the translation model using a relevance feedback provided by 


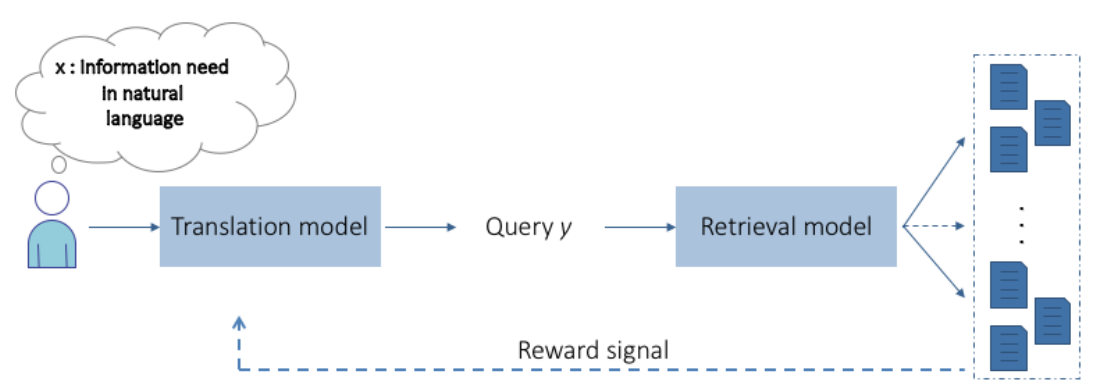

Figure 1: Overview of our reinforcement learning-driven translation model for SOCS

the search engine. It is worth mentioning that there does not exist SOCS-oriented dataset that both aligns users' information needs in NL with keyword-based queries and includes a document collection to perform a retrieval task. To the best of our knowledge, TREC datasets are the only ones expressing such constraint, but the number of NL-query pairs is however limited. To fit with the issue of dealing with large vocabulary and the dataset constraint, we frame the translation model as a word selection one which aims at identifying which words in the NL expression can be used to build the query. Our model is evaluated on two TREC datasets. The obtained results outline the effectiveness of combining reinforcement learning with machine translation models.

The remaining of the paper is organized as follows. Section 2 details our translation model. Section 3 presents the evaluation protocol and results are highlighted in Section 4. The conclusion and perspectives are discussed in Section 5.

\section{Reinforcement learning-driven translation model}

\subsection{Notation and problem formulation}

Our reinforcement learning-driven translation model allows to formulate a user's information need $x$ expressed in NL into a keyword-based query $y$. The user's information need $x$ is a sequence of $n$ words $\left(x=x_{1}, \ldots, x_{i}, \ldots, x_{n}\right)$. To fit with our word selection objective, the query $y$ is modeled as a binary vector $y \in\{0,1\}^{n}$ of size $n$ (namely, the size of the natural language expression $x$ ). Each element $y_{j} \in y$ equals to 1 if word $x_{i} \in x$ exists in query $y$ and 0 otherwise. For example, if we consider the NL as "Identify documents that discuss sick building syndrome or building related illnesses." and the key-words query as "sick building syndrome.", the expected query will be formulated as follows: $y=(0,0,0,0,1,1,1,0,0,0,0)$.

The objective of our model $f_{\theta}$ (with $\theta$ being the parameters of our model) is to estimate the probability $p(y \mid x)$ of generating the binary vector $y$ given the NL expression $x$. Since terms are not independent within the formulation of NL expressions and queries, it makes sense to consider that the selection of a word is conditioned by the sequence of decisions taken on previous words $y_{<i}$. Thus, $P(y \mid x)$ could be written as follows:

$$
p(y \mid x)=\prod_{y_{i} \in y} p\left(y_{i} \mid y_{<i}, x\right)
$$

This probability is first learned using a maximum likelihood estimation (MLE) on the basis of NL-query pairs (Section 2.2). Then, this probability is refined using reinforcement learning techniques (Section 2.3). We end up with the network architecture used in the translation model.

\subsection{Supervised translation model: from NL to queries}

The translation model works as a supervised word selection model aiming at building queries $y$ by using the vocabulary available in NL expressions $x$. To do so, we use a set $D$ of $N$ NL-query pairs $D=\left\{\left(x^{1}, y^{1}\right), \ldots,\left(x^{k}, y^{k}\right), \ldots,\left(x^{N}, y^{N}\right)\right\}$.

The objective of the translation model is to predict whether each word $x_{i}^{k}$ in the NL expression $x^{k}$ is included in the expected query $y^{k}$. In other words, it consists in predicting the probability $p\left(\hat{y}_{i}^{k}=y_{i}^{k} \mid \hat{y}_{<i}^{k}, x^{k}\right)$ that the $i^{\text {th }}$ element $\hat{y}_{i}^{k}$ of vector $\hat{y}^{k}$ is equal to the same element $y_{i}^{k}$ in the original query $y^{k}$ (namely, that $\hat{y}_{i}^{k}=y_{i}^{k}$ ) given the state of previous elements $\hat{y}_{<i}^{k}$ and the NL expression $x^{k}$. This probability $p\left(\hat{y}_{i}^{k}=y_{i}^{k} \mid \hat{y}_{<i}^{k}, x^{k}\right)$ is modeled using a Bernoulli distribution in which parameters are estimated through the probability distribution. 


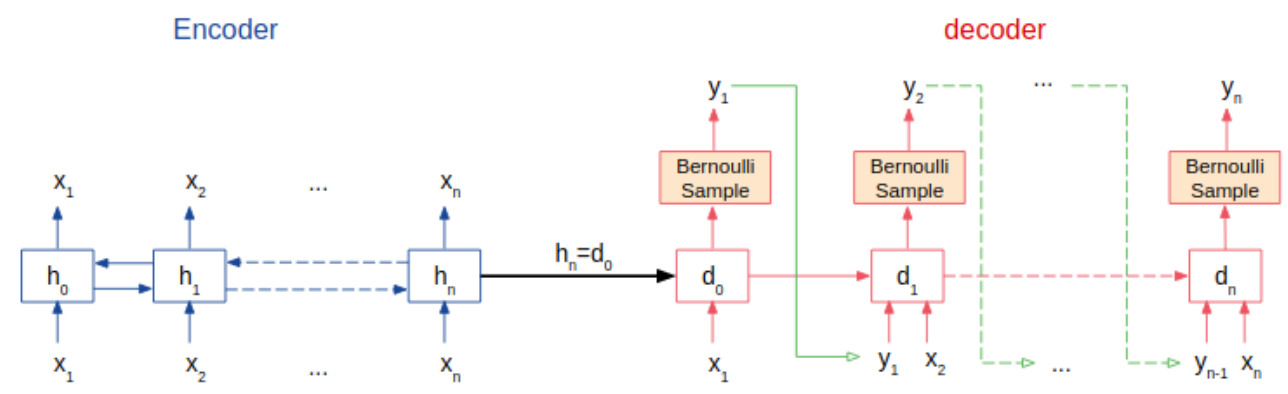

Figure 2: Network architecture of our translation model

Let's define for a NL-query instance $\left(x^{k}, y^{k}\right)$, $f_{\left(\theta, x^{k}\right)}=\sum_{y_{i}^{k} \in y^{k}} \log \left(p\left(\hat{y}_{i}^{k}=y_{i}^{k} \mid \hat{y}_{<i}^{k}, x^{k}\right)\right)$. The translation model is trained by maximizing the following MLE over the set $D$ of NL-query pairs $\left(x_{k}, y_{k}\right)$ :

$$
L_{S M T}=\sum_{\left(x^{k}, y^{k}\right) \in D} \log \left(f\left(\theta, x^{k}\right)\right)
$$

\subsection{Reinforcement learning}

To inject the task objective in the translation model, we consider that the process of query building could be enhanced through reinforcement learning techniques. Therefore, the word selection could be seen as a sequence of choices of selecting word $x_{t}$ at each time step $t$. The choices are rewarded at the end of the selection process by a metric measuring the effectiveness of the query building process within a retrieval task. Particularly, the predicted query $\hat{y}$ obtained from the binary vector $\hat{y}$ is fed to a retrieval model to rank documents. For each NL expression $x$ (and accordingly the associated predicted query $\hat{y}$ ), we dispose of a set $\mathcal{D}_{x}$ of relevant documents (also called ground truth). We note $G T$ the set of $n$ pairs $\left(x ; \mathcal{D}_{x}\right)$. With this in mind, the effectiveness of the obtained ranking could be estimated using an effectiveness-driven metric (e.g., the MAP). Thus, the reward $R$ for a generated query $\hat{y}$ given the relevance feedback pair $\left(x, \mathcal{D}_{x}\right)$ is obtained as follows:

$$
R(\hat{y})=\operatorname{MAP}\left(\hat{y}, \mathcal{D}_{x}\right)
$$

At the end of the selection process, the objective function aims at maximizing the expectation of the search effectiveness over the predicted queries:

$$
L_{R L}(\theta)=\arg \max _{\theta} \mathrm{E}_{\substack{\left(x ; \mathcal{D}_{x}\right) \in G T \\ \hat{y} \sim f_{\theta}(x)}}[R(\hat{y})]
$$

where $\hat{y}$ is given by the translation model $f_{\theta}(x)$. This objective function is maximized using gradient descent techniques (Baxter et al., 1999).

\subsection{Model architecture}

The model is based on an encoder-decoder building a query $\hat{q}$ from the input $x$. Particularly, each element $x_{i}$ of $x$ is modeled through word embeddings $w_{x_{i}}$; resulting in a sequence $w_{x}$ of word embeddings for input $x$. As shown in Figure 2, the encoder is a bi-directional LSTM (Hochreiter and Schmidhuber, 1997) aiming to transform the input sequence $w_{x}$ to its continuous representation $h_{n}$. The decoder is composed of a LSTM in which each word $x_{i}$ is injected to estimate the word selection probability $p\left(y_{i} \mid y_{<i}, x\right)$ using the hidden vector $h_{n}$ learned in the encoder network and the current word $x_{i}$; leading to estimate probability $p\left(y_{i} \mid y_{<i}, x_{i}, h_{n}\right)$.

\section{Protocol design}

\subsection{Datasets}

Since there does not exist yet SOCS-driven datasets including NL-query pairs, we use TREC tracks (namely, Robust 2004 and Web 20002001). In these tracks, query topics include a title, a topic description and a narrative text; the two latter being formulated in natural language. To build query-NL pairs, we use the title to form the set of keyword queries and the description for the set of information needs expressed in NL. An example of a query-NL pair is:

\begin{tabular}{|l|l|}
\hline Title & Lewis and Clark expedition \\
\hline Description & $\begin{array}{l}\text { What are some useful sites containing } \\
\text { information about the historic Lewis } \\
\text { and Clark expedition? }\end{array}$ \\
\hline
\end{tabular}

This NL-query building process results in 350 pairs in total as presented in Table 1.

We are aware that the use of TREC datasets is biased in the sense that it does not exactly fit with the expression of NL information need in the context of conversational systems, but we believe that the description is enough verbose to evaluate the impact of our query building model in this ex- 


\begin{tabular}{|l|l|c|c|c|}
\hline TREC track & collection & pairs & NL length & avg of duplic. word in NL \\
\hline TREC Robust (2004) & disk4-5 & 250 & 15.333 & 1.108 \\
TREC Web (2000 2001) & WT10G & 100 & 11.47 & 0.65 \\
\hline
\end{tabular}

Table 1: Dataset statistics separated per document collections

ploratory work. Further experiments with generated datasets, as done in (Song et al., 2017), will be carried out in the future.

We also analyze the issue of duplicate words into TREC descriptions since it can directly impact the query formulation process based on word selection in the word sequence of TREC descriptions. In practice, this might lead to select several times the same word to build the query, and, therefore, directly impact the retrieval performance. As shown in Table 1, the ratio of duplicate words in TREC descriptions over the whole set of queries is very low (1.1 duplicate words in average in each query for TREC Robust and 0.65 for TREC Web). This suggests that this issue is minor in the used datasets. We, therefore, decided to skip this issue for the moment.

\subsection{Metrics and baselines}

To evaluate our approach, we measure the retrieval effectiveness of the predicted queries. To do so, for each predicted query, we run the BM25 model through an IR system (namely, PyLucene ${ }^{1}$ ) to obtain a document ranking. The latter is evaluated through the MAP metric.

To show the soundness of our approach (namely, translating information needs expressed in NL into queries), we compare our generated queries to scenario NL feeding the natural language information needs (TREC descriptions in our protocol) to the IR retrieval system.

Since the objective of our model is to formulate queries, we also evaluate the effectiveness of original TREC titles (scenario $\mathbf{Q}$ ). This setting rather refers to the oracle that our model must reach.

We mentioned that before training the selection model we transformed each $x$ to its binary representation $y$ based on the presence of the words in the ground truth query. The dataset being slightly biased by this binary modeling, we observed that not all the words existing in the query do exist in $x$. To analyze this bias, we also compare our approach with these binary queries (scenario $\mathbf{Q}$ bin)

\footnotetext{
1'http://lucene.apache.org/pylucene/
}

referring to the projection of queries $\mathbf{Q}$ on the vocabulary available in the NL description.

We also compare our model to a random approach which randomly selects 3 words from $x$ to build queries (scenario Random).

Different variants of our model are also tested:

- SMT which only considers the first component of our model based on a supervised machine translation approach (Section 2.2). This variant could be assimilated to the approach proposed in (Song et al., 2017) in the sense that the machine translation is performed independently of the task objective.

- RL which only considers the reinforcement learning objective function (Section 2.3) without pre-training of the supervised translation model.

- SMT+RL which is our full model in which we start by pre-training the model using the supervised translation model (Section 2.3), and, then, we inject the reward signal in the translation probabilities (Section 2.4).

\subsection{Implementation details}

To transform each word $x_{i}$ to its vector representation $w_{x_{i}}$, we use Fasttext ${ }^{2}$ (Bojanowski et al., 2017) pre-trained word embeddings. The encoder and decoders have one hidden layer with 100 hidden units each.

To train our model, we perform 10 -fold crossvalidation. For the SMT+RL model, we start by a pre-training using the supervised translation model for 100 iterations. The training is then pursued by 1000 iterations while including the reinforcement learning approach. In the latter, the reward, namely the MAP metric, is estimated over document rankings obtained by the BM25 model in PyLucene. We use a minibatch Adam (Kingma and $\mathrm{Ba}, 2014)$ algorithm to pre-train the model and SGD for the reinforcement learning part. Each update is computed after a minibatch of 12 sentences.

\footnotetext{
${ }^{2}$ https://github.com/facebookresearch/ fast Text/
} 


\begin{tabular}{|l|l|l|l|l|}
\hline \multirow{2}{*}{ Baseline } & \multicolumn{2}{|c|}{ TREC Robust(2004) } & \multicolumn{2}{c|}{ TREC Web (2000-2001) } \\
\cline { 2 - 5 } & MAP & $\%$ Chg & MAP & $\%$ Chg \\
\hline NL & 0.08925 & $+15.25 \% * * *$ & 0.15913 & $+12.88 \% *$ \\
\hline Q & 0.09804 & $+4.92 \%$ & 0.16543 & $+8.58 \%$ \\
\hline Q bin & 0.08847 & $+16.26 \% *$ & 0.17402 & $+3.22 \%$ \\
\hline Random & 0.01808 & $+468.91 \% * * *$ & 0.04060 & $+342.44 \% * * *$ \\
\hline SMT & 0.06845 & $+50.27 \% * * *$ & 0.08891 & $+102.04 \% * * *$ \\
\hline RL & 0.08983 & $+14.51 \% * * *$ & 0.16474 & $+9.04 \%$ \\
\hline SMT+RL & $\mathbf{0 . 1 0 2 8 6}$ & & $\mathbf{0 . 1 7 9 6 3}$ & \\
\hline
\end{tabular}

Table 2: Comparative effectiveness analysis of our approach. \%Chg: improvement of SMT+RL over corresponding baselines. Paired t-test significance $*: 0.01<t \leq 0.05 ; * *: 0.001<t \leq 0.01 ; * * *$ : $t \leq 0.001$.

\begin{tabular}{|l|l|l|l|}
\hline NL & Q & Q bin & SMT+RL \\
\hline $\begin{array}{l}\text { what are new methods of producing } \\
\text { steel }\end{array}$ & steel producing & producing steel & $\begin{array}{l}\text { new methods of pro- } \\
\text { ducing steel }\end{array}$ \\
\hline $\begin{array}{l}\text { what are the advantages and or disad- } \\
\text { vantages of tooth implant }\end{array}$ & implant dentistry & implant & $\begin{array}{l}\text { advantages disadvan- } \\
\text { tages tooth implant }\end{array}$ \\
\hline $\begin{array}{l}\text { find documents that discuss the toronto } \\
\text { film festival awards }\end{array}$ & toronto film awards & toronto film awards & $\begin{array}{l}\text { the toronto film festival } \\
\text { awards }\end{array}$ \\
\hline $\begin{array}{l}\text { find documents that give growth rates of } \\
\text { pine trees }\end{array}$ & $\begin{array}{l}\text { where can i find growth } \\
\text { rates for the pine trees }\end{array}$ & growth rates pine trees & $\begin{array}{l}\text { growth rates of pine } \\
\text { trees }\end{array}$ \\
\hline
\end{tabular}

Table 3: Examples of query formulation for $\mathbf{N L}$ queries, the original query $\mathbf{Q}$, the binary version $\mathbf{Q}$ bin of the original query, and our model SMT+RL.

\section{Results}

We present here the effectiveness of our approach aiming at generating queries from users' information needs expressed in NL. In Table 2, we present the retrieval effectiveness (regarding the MAP) of our model and the different baselines (NL, Q, Q bin, Random, SMT, and RL) described in section 3.2. From a general point of view, results highlight that in both datasets, our proposed model SMT+RL outperforms the different baselines with improvements that are generally significant, ranging from $+3.22 \%$ to $+468.91 \%$.

More particularly, the effectiveness analysis allows to draw the following statements:

- The overall performance of the compared approaches generally outperforms the retrieval effectiveness of the NL baseline. For instance, on TREC Robust, queries generated by our model allows to significantly improve the retrieval performance of $+15.25 \%$ regarding information needs expressed in NL (MAP: 0.10286 vs. 0.08925). This result validates the motivation of this work to formulate queries from NL expressions. This is relatively intuitive since NL expressions are verbose by nature and might include non-specific words willing to inject noise in the retrieval process.

- Our approach SMT+RL provides similar re- sults as the $\mathbf{Q}$ and $\mathbf{Q}$ bin. Since the objective function of our model is guided by the initial query $\mathbf{Q}$ transformed in a binary vector ( $\mathbf{Q}$ bin), these baselines could be considered as oracles. We note however that our model obtains higher results (improvements from $+3.22 \%$ to $+16.26 \%$ ) with a significant difference for the $\mathbf{Q}$ bin baseline for TREC Robust. To get a better understanding to what extent our generated queries are different from those used in baselines $\mathbf{Q}$ and $\mathbf{Q}$ bin, we illustrate in Table 3 some examples. While queries in $\mathbf{Q}$ identify the most important words leading to an exploratory query (e.g. "steel productions"), our model SMT+RL provides additional words that precise which facet of the query is concerned (e.g., "new methods of..."), and accordingly improves the ranking of documents.

- Our model SMT+RL is significantly higher than the SMT baseline which converges to a relatively low MAP value ( 0.06845 and 0.08891 for TREC Robust and TREC Web, respectively). This could be explained by the fact that our datasets are very small (250 and 100 NL-query pairs respectively for TREC Robust and TREC Web) and that such machine translation approaches are wellknown to be data hungry. Reinforcement learning techniques could be a solution to overpass this problem since they inject additional information (namely, the reward) in the network learning. 
- The RL baseline achieves relatively good retrieval performances. As we can see from TREC Web, the RL model obtains a MAP of 0.16474 against 0.15913 for the NL baseline. The RL baseline allows approaching the retrieval performances of baselines $\mathbf{Q}$ and $\mathbf{Q}$ bin, although it obtains lower results. This reinforces our intuition that 1) applying machine translation approaches should be driven by the task (retrieval task in our context) and 2) reinforcement learning techniques provide good strategies to build effective queries. The latter statement has also been outlined in previous work (Nogueira and Cho, 2017).

- The comparison of our model SMT+RL regarding SMT and RL baselines outlines that reinforcement learning techniques might be more beneficial when a pre-training is performed. In our context, the pre-training is performed using the SMT model (Section 2.3) which helps the model to be more general and effective before using the reward signal to guide the selection process.

It is worth mentioning that we also trained in preliminary experiments a state of the art translation models such as a generative encoder-decoder RNN with attention mechanism, as done in (Yin et al., 2017; Song et al., 2017). We did not report the results since the model was not able to generalize in the testing phase over new samples from the NL-query dataset used in the training phase. This is probably due to the trade-off between the number of training pairs and the large size of the vocabulary which is not enough represented in different contexts. However, we believe that combining reinforcement learning with attention-mechanism for query-generation is promising. We let this perspective for future work.

\section{Conclusion and future work}

We propose a selection model to transform user's need in NL into a keyword query to increase the retrieval effectiveness in a SOCS context. Our model bridges two lines of work dealing with supervised machine translation and reinforcement learning. Our model has been evaluated using two different TREC datasets and outlines promising results in terms of effectiveness. Our approach has some limitations we plan to overcome in the future. First, our model is framed as a word selection process that could be turned into a generative model. Second, experiments are carried out on small datasets (250 and 100 NL-query pairs) that could be augmented using the evaluation protocol proposed in (Song et al., 2017). In long term, we plan to adapt our model by totally skipping the query formulation step and designing retrieval models dealing with NL expressions.

\section{References}

Eugene Agichtein, Eric Brill, and Susan Dumais. 2006. Improving web search ranking by incorporating user behavior information. In SIGIR '06, pages 19-26.

Jonathan Baxter, Lex Weaver, and Peter Bartlett. 1999. Direct gradient-based reinforcement learning: Ii. gradient ascent algorithms and experiments. Technical report, National University.

Piotr Bojanowski, Edouard Grave, Armand Joulin, and Tomas Mikolov. 2017. Enriching word vectors with subword information. Transactions of the Association for Computational Linguistics, 5:135-146.

Antoine Bordes and Jason Weston. 2016. Learning end-to-end goal-oriented dialog. CoRR, abs/1605.07683.

Mikhail Burtsev, Aleksandr Chuklin, Julia Kiseleva, and Alexey Borisov. 2017. Search-oriented conversational ai (scai). In ICTIR '17, pages 333-334. ACM.

Bhuwan Dhingra, Lihong Li, Xiujun Li, Jianfeng Gao, Yun-Nung Chen, Faisal Ahmed, and Li Deng. 2017. Towards end-to-end reinforcement learning of dialogue agents for information access. In $A C L^{\prime} 17$, pages 484-495.

Sepp Hochreiter and Jürgen Schmidhuber. 1997. Long short-term memory. Neural Comput., 9(8):17351780.

Raphael Hoffmann, Congle Zhang, Xiao Ling, Luke Zettlemoyer, and Daniel S. Weld. 2011. Knowledge-based weak supervision for information extraction of overlapping relations. In $H L T$ ' 11 , pages 541-550.

Thorsten Joachims. 2002. Optimizing Search Engines Using Clickthrough Data. In SIGKDD '02, pages 133-142. ACM.

Hideo Joho, Lawrence Cavedon, Jaime Arguello, Milad Shokouhi, and Filip Radlinski. 2018. Cair'17: First international workshop on conversational approaches to information retrieval at sigir 2017. SIGIR Forum, 51(3):114-121.

Diederik P. Kingma and Jimmy Ba. 2014. Adam: A method for stochastic optimization. CoRR, abs/1412.6980. 
Guillaume Lample, Ludovic Denoyer, and Marc'Aurelio Ranzato. $2017 . \quad$ Unsupervised machine translation using monolingual corpora only. CoRR, abs/1711.00043.

Jiwei Li, Michel Galley, Chris Brockett, Jianfeng Gao, and Bill Dolan. 2016. A diversity-promoting objective function for neural conversation models. In $H L T$ '16, pages 110-119. ACL.

Yankai Lin, Zhiyuan Liu, Maosong Sun, Yang Liu, and Xuan Zhu. 2015. Learning entity and relation embeddings for knowledge graph completion. In $A A A I$, pages 2181-2187. AAAI Press.

Rodrigo Nogueira and Kyunghyun Cho. 2017. Taskoriented query reformulation with reinforcement learning. In SCAI Workshop - ICTIR.

Alan Ritter, Colin Cherry, and William B. Dolan. 2011. Data-driven response generation in social media. In EMNLP' 11

Hyun-Je Song, A-Yeong Kim, and Seong-Bae Park. 2017. Translation of natural language query into keyword query using a rnn encoder-decoder. In SIGIR '17, pages 965-968.

Svitlana Vakulenko, Ilya Markov, and Maarten de Rijke. 2017. Conversational exploratory search via interactive storytelling. In NEUIR SIGIR'17.

Zhuoran Wang and Oliver Lemon. 2013. A simple and generic belief tracking mechanism for the dialog state tracking challenge: On the believability of observed information. In SIGDIAL' 13, page 423-432.

Zi Yin, Keng-hao Chang, and Ruofei Zhang. 2017. Deepprobe: Information directed sequence understanding and chatbot design via recurrent neural networks. In SIGKDD’ 17, pages 2131-2139. 